Bull. Korean Math. Soc. 51 (2014), No. 1, pp. 115-128

http://dx.doi.org/10.4134/BKMS.2014.51.1.115

\title{
ON THE SECOND APPROXIMATE MATSUMOTO METRIC
}

\author{
Akbar Tayebi, Tayebeh Tabatabaeifar, and Esmaeil Peyghan
}

ABstract. In this paper, we study the second approximate Matsumoto metric $F=\alpha+\beta+\beta^{2} / \alpha+\beta^{3} / \alpha^{2}$ on a manifold $M$. We prove that $F$ is of scalar flag curvature and isotropic S-curvature if and only if it is isotropic Berwald metric with almost isotropic flag curvature.

\section{Introduction}

The flag curvature in Finsler geometry is a natural extension of the sectional curvature in Riemannian geometry, which is first introduced by L. Berwald. For a Finsler manifold $(M, F)$, the flag curvature is a function $\mathbf{K}(P, y)$ of tangent planes $P \subset T_{x} M$ and directions $y \in P . F$ is said to be of scalar flag curvature if the flag curvature $\mathbf{K}(P, y)=\mathbf{K}(x, y)$ is independent of flags $P$ associated with any fixed flagpole $y . F$ is called of almost isotropic flag curvature if

$$
\mathbf{K}=\frac{3 c_{x^{m}} y^{m}}{F}+\sigma,
$$

where $c=c(x)$ and $\sigma=\sigma(x)$ are scalar functions on $M$. One of the important problems in Finsler geometry is to characterize Finsler manifolds of almost isotropic flag curvature [10].

To study the geometric properties of a Finsler metric, one also considers non-Riemannian quantities. In Finsler geometry, there are several important non-Riemannian quantities: the Cartan torsion $\mathbf{C}$, the Berwald curvature $\mathbf{B}$, the mean Landsberg curvature $\mathbf{J}$ and S-curvature $\mathbf{S}$, etc. [3, 8, 10, 17]. These are geometric quantities which vanish for Riemnnian metrics.

Among the non-Riemannian quantities, the S-curvature $\mathbf{S}=\mathbf{S}(x, y)$ is closely related to the flag curvature which constructed by Shen for given comparison theorems on Finsler manifolds. A Finsler metric $F$ is called of isotropic $S$ curvature if

$$
\mathbf{S}=(n+1) c F
$$

Received November 18, 2012; Revised March 10, 2013.

2010 Mathematics Subject Classification. 53C60, 53C25.

Key words and phrases. isotropic Berwald curvature, S-curvature, almost isotropic flag curvature.

(C)2014 The Korean Mathematical Society 
for some scalar function $c=c(x)$ on $M$. In [10], it is proved that if a Finsler metric $F$ of scalar flag curvature is of isotropic S-curvature (2), then it has almost isotropic flag curvature (1).

The geodesic curves of a Finsler metric $F=F(x, y)$ on a smooth manifold $M$, are determined by $\ddot{c}^{i}+2 G^{i}(\dot{c})=0$, where the local functions $G^{i}=G^{i}(x, y)$ are called the spray coefficients. A Finsler metric $F$ is called a Berwald metric, if $G^{i}$ are quadratic in $y \in T_{x} M$ for any $x \in M$. A Finsler metric $F$ is said to be isotropic Berwald metric if its Berwald curvature is in the following form

$$
B^{i}{ }_{j k l}=c\left\{F_{y^{j} y^{k}} \delta^{i}{ }_{l}+F_{y^{k} y^{l}} \delta^{i}{ }_{j}+F_{y^{l} y^{j}} \delta^{i}{ }_{k}+F_{y^{j} y^{k} y^{l}} y^{i}\right\},
$$

where $c=c(x)$ is a scalar function on $M[3]$.

As a generalization of Berwald curvature, Bácsó-Matsumoto proposed the notion of Douglas curvature [1]. A Finsler metric is called a Douglas metric if $G^{i}=\frac{1}{2} \Gamma_{j k}^{i}(x) y^{j} y^{k}+P(x, y) y^{i}$.

In order to find explicit examples of Douglas metrics, we consider $(\alpha, \beta)$ metrics. An $(\alpha, \beta)$-metric is a Finsler metric of the form $F:=\alpha \phi\left(\frac{\beta}{\alpha}\right)$, where $\phi=\phi(s)$ is a $C^{\infty}$ on $\left(-b_{0}, b_{0}\right)$ with certain regularity, $\alpha=\sqrt{a_{i j}(x) y^{i} y^{j}}$ is a Riemannian metric and $\beta=b_{i}(x) y^{i}$ is a 1 -form on $M$. This class of metrics is were first introduced by Matsumoto [9]. Among the $(\alpha, \beta)$-metrics, the Matsumoto metric is special and significant metric which constitute a majority of actual research. The Matsumoto metric is expressed as

$$
F=\alpha\left[1+\frac{\beta}{\alpha}+\left(\frac{\beta}{\alpha}\right)^{2}+\left(\frac{\beta}{\alpha}\right)^{3}+\cdots\right]
$$

This metric was introduced by Matsumoto as a realization of Finsler's idea "a slope measure of a mountain with respect to a time measure" [18]. In the Matsumoto metric, the 1 -form $\beta=b_{i} y^{i}$ was originally to be induced by earth gravity. Hence, we could regard $b_{i}(x)$ as the infinitesimals and neglect the infinitesimals of degree of $b_{i}(x)$ more than two $[11,12,13,14,15]$. An approximate Matsumoto metric is a Finsler metric in the following form

$$
F=\alpha\left[\sum_{k=0}^{r}\left(\frac{\beta}{\alpha}\right)^{k}\right],
$$

where $|\beta|<|\alpha|$ (for more information, see [12]). This metric was introduced by Park-Choi in [12]. By definition, the Matsumoto metric is expressed as $\lim _{r \rightarrow \infty} L(\alpha, \beta)=\frac{\alpha^{2}}{\alpha-\beta}$.

In this paper, we consider second approximate Matsumoto metric $F=\alpha+$ $\beta+\frac{\beta^{2}}{\alpha}+\frac{\beta^{3}}{\alpha^{2}}$ with some non-Riemannian curvature properties and prove the following.

Theorem 1.1. Let $F=\alpha+\beta+\frac{\beta^{2}}{\alpha}+\frac{\beta^{3}}{\alpha^{2}}$ be a non-Riemannian second approximate Matsumoto metric on a manifold $M$ of dimension $n$. Then $F$ is of scaler flag curvature with isotropic S-curvature (2), if and only if it has isotropic 
Berwald curvature (3) with almost isotropic flag curvature (1). In this case, $F$ must be locally Minkowskian.

\section{Preliminaries}

Let $M$ be a $n$-dimensional $C^{\infty}$ manifold. Denote by $T_{x} M$ the tangent space at $x \in M$, by $T M=\cup_{x \in M} T_{x} M$ the tangent bundle of $M$, and by $T M_{0}=$ $T M \backslash\{0\}$ the slit tangent bundle on $M$. A Finsler metric on $M$ is a function $F: T M \rightarrow[0, \infty)$ which has the following properties:

(i) $F$ is $C^{\infty}$ on $T M_{0}$;

(ii) $F$ is positively 1-homogeneous on the fibers of tangent bundle $T M$;

(iii) for each $y \in T_{x} M$, the following quadratic form $\mathbf{g}_{y}$ on $T_{x} M$ is positive definite,

$$
\mathbf{g}_{y}(u, v):=\left.\frac{1}{2} \frac{\partial^{2}}{\partial s \partial t}\left[F^{2}(y+s u+t v)\right]\right|_{s, t=0}, \quad u, v \in T_{x} M
$$

Let $x \in M$ and $F_{x}:=\left.F\right|_{T_{x} M}$. To measure the non-Euclidean feature of $F_{x}$, define $\mathbf{C}_{y}: T_{x} M \otimes T_{x} M \otimes T_{x} M \rightarrow \mathbb{R}$ by

$$
\mathbf{C}_{y}(u, v, w):=\left.\frac{1}{2} \frac{d}{d t}\left[\mathbf{g}_{y+t w}(u, v)\right]\right|_{t=0}, \quad u, v, w \in T_{x} M .
$$

The family $\mathbf{C}:=\left\{\mathbf{C}_{y}\right\}_{y \in T M_{0}}$ is called the Cartan torsion. It is well known that $\mathbf{C}=0$ if and only if $F$ is Riemannian [16]. For $y \in T_{x} M_{0}$, define mean Cartan torsion $\mathbf{I}_{y}$ by $\mathbf{I}_{y}(u):=I_{i}(y) u^{i}$, where $I_{i}:=g^{j k} C_{i j k}$. By Diecke Theorem, $F$ is Riemannian if and only if $\mathbf{I}_{y}=0$.

The horizontal covariant derivatives of $\mathbf{I}$ along geodesics give rise to the mean Landsberg curvature $\mathbf{J}_{y}(u):=J_{i}(y) u^{i}$, where $J_{i}:=I_{i \mid s} y^{s}$. A Finsler metric is said to be weakly Landsbergian if $\mathbf{J}=0$.

Given a Finsler manifold $(M, F)$, then a global vector field $\mathbf{G}$ is induced by $F$ on $T M_{0}$, which in a standard coordinate $\left(x^{i}, y^{i}\right)$ for $T M_{0}$ is given by $\mathbf{G}=y^{i} \frac{\partial}{\partial x^{i}}-2 G^{i}(x, y) \frac{\partial}{\partial y^{i}}$, where

$$
G^{i}:=\frac{1}{4} g^{i l}\left[\frac{\partial^{2}\left(F^{2}\right)}{\partial x^{k} \partial y^{l}} y^{k}-\frac{\partial\left(F^{2}\right)}{\partial x^{l}}\right], \quad y \in T_{x} M .
$$

The $\mathbf{G}$ is called the spray associated to $(M, F)$. In local coordinates, a curve $c(t)$ is a geodesic if and only if its coordinates $\left(c^{i}(t)\right)$ satisfy $\ddot{c}^{i}+2 G^{i}(\dot{c})=0$.

For a tangent vector $y \in T_{x} M_{0}$, define $\mathbf{B}_{y}: T_{x} M \otimes T_{x} M \otimes T_{x} M \rightarrow T_{x} M$ and $\mathbf{E}_{y}: T_{x} M \otimes T_{x} M \rightarrow \mathbb{R}$ by $\mathbf{B}_{y}(u, v, w):=\left.B^{i}{ }_{j k l}(y) u^{j} v^{k} w^{l} \frac{\partial}{\partial x^{i}}\right|_{x}$ and $\mathbf{E}_{y}(u, v):=$ $E_{j k}(y) u^{j} v^{k}$ where

$$
B_{j k l}^{i}:=\frac{\partial^{3} G^{i}}{\partial y^{j} \partial y^{k} \partial y^{l}}, \quad E_{j k}:=\frac{1}{2} B_{j k m}^{m} .
$$

The $\mathbf{B}$ and $\mathbf{E}$ are called the Berwald curvature and mean Berwald curvature, respectively. Then $F$ is called a Berwald metric and weakly Berwald metric if $\mathbf{B}=\mathbf{0}$ and $\mathbf{E}=\mathbf{0}$, respectively. 
A Finsler metric $F$ is said to be isotropic mean Berwald metric if its mean Berwald curvature is in the following form

$$
E_{i j}=\frac{n+1}{2 F} c h_{i j}
$$

where $c=c(x)$ is a scalar function on $M$ and $h_{i j}$ is the angular metric [3].

Define $\mathbf{D}_{y}: T_{x} M \otimes T_{x} M \otimes T_{x} M \rightarrow T_{x} M$ by $\mathbf{D}_{y}(u, v, w):=\left.D^{i}{ }_{j k l}(y) u^{i} v^{j} w^{k} \frac{\partial}{\partial x^{i}}\right|_{x}$ where

$$
D^{i}{ }_{j k l}:=B^{i}{ }_{j k l}-\frac{2}{n+1}\left\{E_{j k} \delta_{l}^{i}+E_{j l} \delta_{k}^{i}+E_{k l} \delta_{j}^{i}+E_{j k, l} y^{i}\right\} .
$$

We call $\mathbf{D}:=\left\{\mathbf{D}_{y}\right\}_{y \in T M_{0}}$ the Douglas curvature. A Finsler metric with $\mathbf{D}=0$ is called a Douglas metric. The notion of Douglas metrics was proposed by Bácsó-Matsumoto as a generalization of Berwald metrics [1].

For a Finsler metric $F$ on an $n$-dimensional manifold $M$, the BusemannHausdorff volume form $d V_{F}=\sigma_{F}(x) d x^{1} \cdots d x^{n}$ is defined by

$$
\sigma_{F}(x):=\frac{\operatorname{Vol}\left(\mathbb{B}^{n}(1)\right)}{\operatorname{Vol}\left\{\left(y^{i}\right) \in R^{n} \mid F\left(\left.y^{i} \frac{\partial}{\partial x^{i}}\right|_{x}\right)<1\right\}} .
$$

In general, the local scalar function $\sigma_{F}(x)$ can not be expressed in terms of elementary functions, even $F$ is locally expressed by elementary functions. Let $G^{i}$ denote the geodesic coefficients of $F$ in the same local coordinate system. The S-curvature can be defined by

$$
\mathbf{S}(\mathbf{y}):=\frac{\partial G^{i}}{\partial y^{i}}(x, y)-y^{i} \frac{\partial}{\partial x^{i}}\left[\ln \sigma_{F}(x)\right],
$$

where $\mathbf{y}=\left.y^{i} \frac{\partial}{\partial x^{i}}\right|_{x} \in T_{x} M$. It is proved that $\mathbf{S}=0$ if $F$ is a Berwald metric. There are many non-Berwald metrics satisfying $\mathbf{S}=0$. $\mathbf{S}$ said to be isotropic if there is a scalar functions $c(x)$ on $M$ such that $\mathbf{S}=(n+1) c(x) F$.

The Riemann curvature $\mathbf{R}_{y}=\left.R_{k}^{i} d x^{k} \otimes \frac{\partial}{\partial x^{i}}\right|_{x}: T_{x} M \rightarrow T_{x} M$ is a family of linear maps on tangent spaces, defined by

$$
R_{k}^{i}=2 \frac{\partial G^{i}}{\partial x^{k}}-y^{j} \frac{\partial^{2} G^{i}}{\partial x^{j} \partial y^{k}}+2 G^{j} \frac{\partial^{2} G^{i}}{\partial y^{j} \partial y^{k}}-\frac{\partial G^{i}}{\partial y^{j}} \frac{\partial G^{j}}{\partial y^{k}} .
$$

For a flag $P=\operatorname{span}\{y, u\} \subset T_{x} M$ with flagpole $y$, the flag curvature $\mathbf{K}=$ $\mathbf{K}(P, y)$ is defined by

$$
\mathbf{K}(P, y):=\frac{\mathbf{g}_{y}\left(u, \mathbf{R}_{y}(u)\right)}{\mathbf{g}_{y}(y, y) \mathbf{g}_{y}(u, u)-\mathbf{g}_{y}(y, u)^{2}} .
$$

We say that a Finsler metric $F$ is of scalar curvature if for any $y \in T_{x} M$, the flag curvature $\mathbf{K}=\mathbf{K}(x, y)$ is a scalar function on the slit tangent bundle $T M_{0}$. In this case, for some scalar function $\mathbf{K}$ on $T M_{0}$ the Riemann curvature is in the following form

$$
R_{k}^{i}=\mathbf{K} F^{2}\left\{\delta_{k}^{i}-F^{-1} F_{y^{k}} y^{i}\right\} .
$$

If $\mathbf{K}=$ constant, then $F$ is said to be of constant flag curvature. A Finsler metric $F$ is called isotropic flag curvature, if $\mathbf{K}=\mathbf{K}(x)$. 


\section{Proof of Theorem 1.1}

Let $F=\alpha \phi(s), s=\frac{\beta}{\alpha}$ be an $(\alpha, \beta)$-metric, where $\phi=\phi(s)$ is a $C^{\infty}$ on $\left(-b_{0}, b_{0}\right)$ with certain regularity, $\alpha=\sqrt{a_{i j}(x) y^{i} y^{j}}$ is a Riemannian metric and $\beta=b_{i}(x) y^{i}$ is a 1 -form on a manifold $M$. Let

$$
\begin{gathered}
r_{i j}:=\frac{1}{2}\left[b_{i \mid j}+b_{j \mid i}\right], \quad s_{i j}:=\frac{1}{2}\left[b_{i \mid j}-b_{j \mid i}\right] . \\
r_{j}:=b^{i} r_{i j}, \quad s_{j}:=b^{i} s_{i j},
\end{gathered}
$$

where $b_{i \mid j}$ denote the coefficients of the covariant derivative of $\beta$ with respect to $\alpha$. Let

$$
r_{i 0}:=r_{i j} y^{j}, \quad s_{i 0}:=s_{i j} y^{j}, \quad r_{0}:=r_{j} y^{j}, \quad s_{0}:=s_{j} y^{j}
$$

Put

$$
\begin{aligned}
Q & =\frac{\phi^{\prime}}{\phi-s \phi}, \\
\Theta & =\frac{\phi \phi^{\prime}-s\left(\phi \phi^{\prime \prime}+\phi^{\prime 2}\right)}{2 \phi\left[\left(\phi-s \phi^{\prime}\right)+\left(b^{2}-s^{2}\right) \phi^{\prime \prime}\right]} \\
\Psi & =\frac{\phi^{\prime \prime}}{2\left[\left(\phi-s \phi^{\prime}\right)+\left(b^{2}-s^{2}\right) \phi^{\prime \prime}\right]} .
\end{aligned}
$$

Then the $S$-curvature is given by

$$
\begin{aligned}
\mathbf{S}=[ & \left.Q^{\prime}-2 \Psi Q s-2(\Psi Q)^{\prime}\left(b^{2}-s^{2}\right)-2(n+1) Q \Theta+2 \lambda\right] s_{0} \\
& +2(\Psi+\lambda) s_{0}+\alpha^{-1}\left[\left(b^{2}-s^{2}\right) \Psi^{\prime}+(n+1) \Theta\right] r_{00} .
\end{aligned}
$$

Let us put

$$
\begin{aligned}
& \Delta:=1+s Q+\left(b^{2}-s^{2}\right) Q^{\prime}, \\
& \Phi:=-(n \Delta+1+s Q)\left(Q-s Q^{\prime}\right)-\left(b^{2}-s^{2}\right)(1+s Q) Q^{\prime \prime} .
\end{aligned}
$$

In [5], Cheng-Shen characterize $(\alpha, \beta)$-metrics with isotropic S-curvature.

Lemma $3.1([5])$. Let $F=\alpha \phi(\beta / \alpha)$ be an $(\alpha, \beta)$-metric on an $n$-manifold. Then, $F$ is of isotropic $S$-curvature $\mathbf{S}=(n+1) c F$, if and only if one of the following holds

(i) $\beta$ satisfies

$$
r_{i j}=\varepsilon\left\{b^{2} a_{i j}-b_{i} b_{j}\right\}, \quad s_{j}=0,
$$

where $\varepsilon=\varepsilon(x)$ is a scalar function, and $\phi=\phi(s)$ satisfies

$$
\Phi=-2(n+1) k \frac{\phi \Delta^{2}}{b^{2}-s^{2}},
$$

where $k$ is a constant. In this case, $c=k \epsilon$.

(ii) $\beta$ satisfies

$$
r_{i j}=0, \quad s_{j}=0 \text {. }
$$


In this case, $c=0$.

Let

$$
\begin{aligned}
\Psi_{1} & :=\sqrt{b^{2}-s^{2}} \Delta^{\frac{1}{2}}\left[\frac{\sqrt{b^{2}-s^{2}} \Phi}{\Delta^{\frac{3}{2}}}\right]^{\prime}, \\
\Psi_{2} & :=2(n+1)\left(Q-s Q^{\prime}\right)+3 \frac{\Phi}{\Delta}, \\
\theta & :=\frac{Q-s Q^{\prime}}{2 \Delta} .
\end{aligned}
$$

Then the formula for the mean Cartan torsion of an $(\alpha, \beta)$-metric is given by following

$$
\begin{aligned}
I_{i} & =\frac{1}{2} \frac{\partial}{\partial y^{i}}\left[(n+1) \frac{\phi^{\prime}}{\phi}-(n-2) \frac{s \phi^{\prime \prime}}{\phi-s \phi^{\prime}}-\frac{3 s \phi^{\prime \prime}-\left(b^{2}-s^{2}\right) \phi^{\prime \prime \prime}}{\left(\phi-s \phi^{\prime}\right)+\left(b^{2}-s^{2}\right) \phi^{\prime \prime}}\right] \\
& =-\frac{\Phi\left(\phi-s \phi^{\prime}\right)}{2 \Delta \phi \alpha^{2}}\left(\alpha b_{i}-s y_{i}\right) .
\end{aligned}
$$

In [6], it is proved that the condition $\Phi=0$ characterizes the Riemannian metrics among $(\alpha, \beta)$-metrics. Hence, in the continue, we suppose that $\Phi \neq 0$.

Let $G^{i}=G^{i}(x, y)$ and $\bar{G}_{\alpha}^{i}=\bar{G}_{\alpha}^{i}(x, y)$ denote the coefficients of $F$ and $\alpha$ respectively in the same coordinate system. By definition, we have

$$
G^{i}=\bar{G}_{\alpha}^{i}+P y^{i}+Q^{i}
$$

where

$$
\begin{aligned}
P & :=\alpha^{-1} \Theta\left[-2 Q \alpha s_{0}+r_{00}\right] \\
Q^{i} & :=\alpha Q s_{0}^{i}+\Psi\left[-2 Q \alpha s_{0}+r_{00}\right] b^{i} .
\end{aligned}
$$

Simplifying (13) yields the following

$$
G^{i}=\bar{G}_{\alpha}^{i}+\alpha Q s_{0}^{i}+\theta\left(-2 \alpha Q s_{0}+r_{00}\right)\left[\frac{y^{i}}{\alpha}+\frac{Q^{\prime}}{Q-s Q^{\prime}} b^{i}\right] .
$$

Clearly, if $\beta$ is parallel with respect to $\alpha\left(r_{i j}=0\right.$ and $\left.s_{i j}=0\right)$, then $P=0$ and $Q^{i}=0$. In this case, $G^{i}=\bar{G}_{\alpha}^{i}$ are quadratic in $y$, and $F$ is a Berwald metric.

For an $(\alpha, \beta)$-metric $F=\alpha \phi(s)$, the mean Landsberg curvature is given by

$$
\begin{aligned}
& J_{i}=-\frac{1}{2 \Delta \alpha^{4}}\left[\frac{2 \alpha^{2}}{b^{2}-s^{2}}\left[\frac{\Phi}{\Delta}+(n+1)\left(Q-s Q^{\prime}\right)\right]\left(r_{0}+s_{0}\right) h_{i}\right. \\
&+\frac{\alpha}{b^{2}-s^{2}}\left(\Psi_{1}+s \frac{\Phi}{\Delta}\right)\left(r_{00}-2 \alpha Q s_{0}\right) h_{i}+\alpha\left[-\alpha Q^{\prime} s_{0} h_{i}+\alpha Q\left(\alpha^{2} s_{i}-y_{i} s_{0}\right)\right. \\
&\left.\left.(15)+\alpha^{2} \Delta s_{i 0}+\alpha^{2}\left(r_{i 0}-2 \alpha Q s_{i}\right)-\left(r_{00}-2 \alpha Q s_{0}\right) y_{i}\right] \frac{\Phi}{\Delta}\right] .
\end{aligned}
$$


Contracting (15) with $b^{i}=a^{i m} b_{m}$ yields

$$
\bar{J}:=J_{i} b^{i}=-\frac{1}{2 \Delta \alpha^{2}}\left[\Psi_{1}\left(r_{00}-2 \alpha Q s_{0}\right)+\alpha \Psi_{2}\left(r_{0}+s_{0}\right)\right] .
$$

The horizontal covariant derivatives $J_{i ; m}$ and $J_{i \mid m}$ of $J_{i}$ with respect to $F$ and $\alpha$, respectively, are given by

Then we have

$$
\begin{aligned}
& J_{i ; m}=\frac{\partial J_{i}}{\partial x^{m}}-J_{l} \Gamma_{i m}^{l}-\frac{\partial J_{i}}{\partial y^{l}} N_{m}^{l} \\
& J_{i \mid m}=\frac{\partial J_{i}}{\partial x^{m}}-J_{l} \bar{\Gamma}_{i m}^{l}-\frac{\partial J_{i}}{\partial y^{l}} \bar{N}_{m}^{l} .
\end{aligned}
$$

$$
J_{i ; m} y^{m}=J_{i \mid m} y^{m}-J_{l}\left(N_{i}^{l}-\bar{N}_{i}^{l}\right)-2 \frac{\partial J_{i}}{\partial y^{l}}\left(G^{l}-\bar{G}^{l}\right) .
$$

Let $F$ be a Finsler metric of scalar flag curvature K. By Akbar-Zadeh's theorem it satisfies following

$$
A_{i j k ; s ; m} y^{s} y^{m}+\mathbf{K} F^{2} A_{i j k}+\frac{F^{2}}{3}\left[h_{i j} \mathbf{K}_{k}+h_{j k} \mathbf{K}_{j}+h_{k i} \mathbf{K}_{j}\right]=0
$$

where $A_{i j k}=F C_{i j k}$ is the Cartan torsion and $\mathbf{K}_{i}=\frac{\partial \mathbf{K}}{\partial y^{i}}$ [2]. Contracting (18) with $g^{i j}$ yields

$$
J_{i ; m} y^{m}+\mathbf{K} F^{2} I_{i}+\frac{n+1}{3} F^{2} \mathbf{K}_{i}=0 .
$$

By (17) and (19), for an $(\alpha, \beta)$-metric $F=\alpha \phi(s)$ of constant flag curvature $\mathbf{K}$, the following holds

$$
J_{i \mid m}-J_{l} \frac{\partial\left(G^{l}-\bar{G}^{l}\right)}{\partial y^{i}} b^{i}-2 \frac{\partial \bar{J}}{\partial y^{l}}\left(G^{l}-\bar{G}^{l}\right) \mathbf{K} \alpha^{2} \phi^{2} I_{i}=0 .
$$

Contracting (20) with $b^{i}$ implies that

(21) $\bar{J}_{\mid m} y^{m}-J_{i} a^{i k} b_{k \mid m} y^{m}-J_{l} \frac{\partial\left(G^{l}-\bar{G}^{l}\right)}{\partial y^{i}} b^{i}-2 \frac{\partial \bar{J}}{\partial y^{l}}\left(G^{l}-\bar{G}^{l}\right)+\boldsymbol{K} \alpha^{2} \phi^{2} I_{i} b^{i}=0$.

There exists a relation between mean Berwald curvature $\mathbf{E}$ and the $\mathrm{S}$ curvature S. Indeed, taking twice vertical covariant derivatives of the Scurvature gives rise the $E$-curvature. It is easy to see that, every Finsler metric of isotropic S-curvature (2) is of isotropic mean Berwald curvature (5). Now, is the equation $\mathbf{S}=(n+1) c F$ equivalent to the equation $\mathbf{E}=\frac{n+1}{2} c F^{-1} \mathbf{h}$ ?

Recently, Cheng-Shen prove that a Randers metric $F=\alpha+\beta$ is of isotropic $S$-curvature if and only if it is of isotropic E-curvature [4]. Then, Chun-HuanCheng extend this equivalency to the Finsler metric $F=\alpha^{-m}(\alpha+\beta)^{m+1}$ for every real constant $m$, including Randers metric [20]. In [7], Cui extend their result and show that for the Matsumoto metric $F=\frac{\alpha^{2}}{\alpha-\beta}$ and the special $(\alpha, \beta)$-metric $F=\alpha+\epsilon \beta+\kappa\left(\beta^{2} / \alpha\right)(\kappa \neq 0)$, these notions are equivalent.

To prove Theorem 1.1, we need the following. 
Proposition 3.2. Let $F=\alpha+\beta+\frac{\beta^{2}}{\alpha}+\frac{\beta^{3}}{\alpha^{2}}$ be a second approximate Matsumoto metric on a manifold $M$ of dimension $n$. Then the following are equivalent

(i) $F$ has isotropic $S$-curvature, $\mathbf{S}=(n+1) c(x) F$;

(ii) $F$ has isotropic mean Berwald curvature, $\mathbf{E}=\frac{n+1}{2} c(x) F^{-1} \mathbf{h}$;

where $c=c(x)$ is a scalar function on the manifold $M$. In this case, $\mathbf{S}=0$. Then $\beta$ is a Killing 1-form with constant length with respect to $\alpha$, that is, $r_{00}=0$.

Proof. (i) $\Rightarrow$ (ii) is obvious. Conversely, suppose that $F$ has isotropic mean Berwald curvature, $\mathbf{E}=\frac{(n+1)}{2} c(x) F^{-1} \mathbf{h}$. Then we have

$$
\mathbf{S}=(n+1)[c F+\eta],
$$

where $\eta=\eta_{i}(x) y^{i}$ is a 1 -form on $M$. For the second approximate Matsumoto metric, (6) reduces to following

$$
\begin{aligned}
& Q=-\frac{1+2 s+3 s^{2}}{-1+s^{2}+2 s^{3}}, \\
& \Theta=\frac{1}{2} \frac{1-6 s^{2}-12 s^{3}-15 s^{4}-12 s^{5}}{\left(1+s+s^{2}+s^{3}\right)\left(1-3 s^{2}-8 s^{3}+2 b^{2}+6 b^{2} s\right)}, \\
& \Psi=\frac{1+3 s}{\left(1-3 s^{2}-8 s^{3}+2 b^{2}+6 b^{2} s\right)} .
\end{aligned}
$$

By substituting (22) and (23) in (7), we have

$$
\begin{aligned}
\mathbf{S}= & {\left[\frac{2(1+3 s)\left(1+s+s^{2}+s^{3}\right)}{\left(-1+s^{2}+2 s^{3}\right)^{2}}+\frac{2(1+3 s)\left(1+2 s+3 s^{2}\right) s}{\left(1-3 s^{2}-8 s^{3}+2 b^{2}+6 b^{2} s\right)\left(-1+s^{2}+2 s^{3}\right)}\right.} \\
& -\frac{2\left(5+26 s+77 s^{2}+88 s^{3}-61 s^{4}-430 s^{5}-805 s^{6}+4 b^{2}+40 b^{2} s+148 b^{2} s^{2}\right)\left(b^{2}-s^{2}\right)}{\left(1-3 s^{2}-8 s^{3}+2 b^{2}+6 b^{2} s\right)^{2}\left(-1+s^{2}+2 s^{3}\right)^{2}} \\
& -\frac{2\left(256 s^{3} b^{2}+252 s^{4} b^{2}+216 s^{5} b^{2}+108 s^{6} b^{2}-828 s^{7}-432 s^{8}\right)\left(b^{2}-s^{2}\right)}{\left(1-3 s^{2}-8 s^{3}+2 b^{2}+6 b^{2} s\right)^{2}\left(-1+s^{2}+2 s^{3}\right)^{2}} \\
& \left.+\frac{(n+1)\left(1+2 s+3 s^{2}\right)\left(1-6 s^{2}-12 s^{3}-15 s^{4}-12 s^{5}\right)}{\left(-1+s^{2}+2 s^{3}\right)\left(1+s+s^{2}+s^{3}\right)\left(1-3 s^{2}-8 s^{3}+2 b^{2}+6 b^{2} s\right)}+2 \lambda\right] s_{0} \\
& +2\left[\frac{(1+3 s)}{1-3 s^{2}-8 s^{3}+2 b^{2}+6 b^{2} s}+\lambda\right]+\left[\frac{3\left(b^{2}-s^{2}\right)\left(1+11 s^{2}+16 s^{3}+2 s\right)}{\alpha\left(1-3 s^{2}-8 s^{3}+2 b^{2}+6 b^{2} s\right)^{2}}\right] r_{00} \\
& +\left[\frac{(n+1)\left(1-6 s^{2}-12 s^{3}-15 s^{4}-12 s^{5}\right)}{2 \alpha\left(1+s+s^{2}+s^{3}\right)\left(1-3 s^{2}-8 s^{3}+2 b^{2}+6 b^{2} s\right)}\right] r_{00} \\
= & (n+1)\left[c \alpha\left(1+s+s^{2}+s^{3}\right)+\eta\right] .
\end{aligned}
$$

Multiplying (24) with $\left(-1+s^{2}+2 s^{3}\right)\left(1+s+s^{2}+s^{3}\right)\left(1-3 s^{2}-8 s^{3}+2 b^{2}+6 b^{2} s\right)^{2} \alpha^{14}$ implies that

$$
M_{1}+M_{2} \alpha^{2}+M_{3} \alpha^{4}+M_{4} \alpha^{6}+M_{5} \alpha^{8}+M_{6} \alpha^{10}+M_{7} \alpha^{12}+M_{8} \alpha^{14}
$$




$$
\begin{array}{r}
+\alpha\left[M_{9}+M_{10} \alpha^{2}+M_{11} \alpha^{4}+M_{12} \alpha^{6}+M_{13} \alpha^{8}+M_{14} \alpha^{10}\right. \\
\left.+M_{15} \alpha^{12}+M_{16} \alpha^{14}\right]=0,
\end{array}
$$

where

$$
\begin{aligned}
M_{1}:= & -128(n+1) c \beta^{15}, \\
M_{2}:=2 & {\left[(n+1)\left[\left(-385+96 b^{2}\right) c \beta^{2}-64 \eta \beta\right]+128 \lambda\left(r_{0}+s_{0}\right) \beta+48 n r_{00}\right] \beta^{11}, } \\
M_{3}:= & -\left[(n+1)\left[4\left(-283 b^{2}+243+18 b^{4}\right) c \beta^{2}-6\left(32 b^{2}-59\right) \eta \beta\right]\right. \\
& +\left[12\left(-59+32 b^{2}\right) \lambda\left(r_{0}+s_{0}\right)-96\left((3 n-1) s_{0}-r_{0}\right] \beta\right. \\
& \left.+3\left(-24 b^{2}+36-219 n+72 n b^{2}\right) r_{00}\right] \beta^{9}, \\
M_{4}:= & (n+1)\left[-2\left(29-738 b^{2}+208 b^{4}\right) c \beta^{2}-3\left(-172 b^{2}+21+24 b^{4}\right) \eta \beta\right] \\
& +\left(-159 n b^{2}-132-39 n+96 b^{2}\right) r_{00} \\
& -3\left(-48 b^{2}+123+72 n b^{2}-277 n\right) s_{0} \beta \\
& \left.+3\left(24 b^{2}+86\right) r_{0} \beta+6 \lambda\left(-172 b^{2}+21+24 b^{4}\right)\left(s_{0}+r_{0}\right) \beta\right] \beta^{7}, \\
M_{5}:= & (n+1)\left[-8\left(-27 b^{2}+70 b^{4}-41\right) c \beta^{2}-4\left(47 b^{4}-40-32 b^{2}\right) \eta \beta\right] \\
& +\left(15 n b^{2}+20-55 n+108 b^{2}\right) r_{00}+8 \lambda\left(47 b^{4}-40-32 b^{2}\right)\left(r_{0}+s_{0}\right) \beta \\
& \left.-2\left[\left(-262 b^{2}+278+303 n b^{2}-147 n\right) s_{0}-\left(94 b^{2}+32\right) r_{0}\right] \beta\right] \beta^{5} \\
M_{6}:=2 & {\left[(n+1)\left[-2(4 b+1)(4 b-1)\left(4 b^{2}+13\right) c \beta^{2}-10\left(1+20 b^{2}+6 b^{4}\right) \eta \beta\right]\right.} \\
& +\left(11 n-40 b^{2}+35 n b^{2}+20\right) r_{00}+20 \lambda\left(1+20 b^{2}+6 b^{4}\right)\left(r_{0}+s_{0}\right) \beta \\
& \left.-2\left(32 n+51+129 n b^{2}-294 b^{2}\right) s_{0} \beta-\left(30 b^{2}-50\right) r_{0} \beta\right] \beta^{3}, \\
M_{7}:= & -\left[(n+1)\left[-4\left(-17 b^{2}-7+30 b^{4}\right) c \beta^{2}-6\left(-1+10 b^{4}\right) \beta\right]\right. \\
& +(3 n+12) b^{2} r_{00} \\
& \left.-12 \lambda\left(1-10 b^{4}\right)\left(s_{0}+r_{0}\right)-6\left[\left(n b^{2}-n-2+14 b^{2}\right) s_{0}-10 b^{2} r_{0}\right] \beta\right] \beta, \\
M_{8}:=4 & {\left[(n+1)\left[2\left(1+2 b^{2}\right)\left(8 b^{2}+1\right) c \beta+\left(1+2 b^{2}\right)^{2} \eta\right]\right.} \\
& -2 \lambda\left(1+2 b^{2}\right)^{2}\left(s_{0}+r_{0}\right) \\
& +\left[-57 n b^{2}-64(n+1) b^{4}-8 n-55 b^{2}+6\right] r_{00}-\left(2-4 b^{2}\right) r_{0} \\
& \left.+\left[n+(4+2 n) b^{2}-1\right] s_{0}\right], \\
M_{9}:= & -416(n+1) c \beta^{14}, \\
& {[n}
\end{aligned}
$$




$$
\begin{aligned}
& M_{10}:=\left[(n+1)\left[\left(-1037+616 b^{2}\right) c \beta^{2}-288 \eta \beta\right]\right. \\
& \left.+576 \lambda\left(r_{0}+s_{0}\right) \beta+(204 n-6) r_{00}\right] \beta^{12}, \\
& M_{11}:=-\frac{1}{2}\left[(n+1)\left[8\left(57 b^{4}-385 b^{2}+143\right) c \beta^{2}-2\left(424 b^{2}-267\right) \eta \beta\right]\right. \\
& +\left[4 \lambda\left(424 b^{2}-267\right)\left(r_{0}+s_{0}\right)-4(-115+330 n) s_{0}+1320 r_{0}\right] \beta \\
& \left.+\left(300 n b^{2}+249-189 n-120 b^{2}\right) r_{00}\right] \beta^{8}, \\
& M_{12}:=4\left[(n+1)\left[-\left(572 b^{4}-932 b^{2}-275\right) c \beta^{2}-4\left(39 b^{4}-28-102 b^{2}\right) \eta \beta\right]\right. \\
& -75 n b^{2}-62-89 n+144 b^{2} r_{00}+8 \lambda\left(39 b^{4}-28-102 b^{2}\right)\left(r_{0}+s_{0}\right) \beta \\
& \left.-6\left[\left(-58 b^{2}+100+81 n b^{2}-109 n\right) s_{0}-\left(26 b^{2}+34\right) r_{0}\right] \beta\right] \beta^{6}, \\
& M_{13}:=\left[(n+1)\left[-8\left(-24+35 b^{2}+47 b^{4}\right) c \beta^{2}-6\left(26 b^{4}-11+20 b^{2}\right) \eta \beta\right]\right. \\
& +\left(51 n b^{2}+33-6 n+24 b^{2}\right) r_{00}+12 \lambda\left(26 b^{4}-11+20 b^{2}\right)\left(s_{0}+r_{0}\right) \\
& \left.-6\left(-118 b^{2}+54+83 n b^{2}-3 n\right) s_{0} \beta+6\left(26 b^{2}-10\right) r_{0} \beta\right] \beta^{4} \text {, } \\
& M_{14}:=-\left[( n + 1 ) \left[-\left(56 b^{4}-272 b^{2}-39\right) c \beta^{2}-4\left(7(n+1) b^{4}\right.\right.\right. \\
& \left.\left.-6(1+n)-22 n b^{2}\right) \eta \beta\right] \\
& +\left(-7 n b^{2}-8-5 n+32 b^{2}\right) r_{00}+8 \lambda\left(7 b^{4}-6-22 b^{2}\right)\left(r_{0}+s_{0}\right) \beta \\
& \left.+2\left(-154 b^{2}+12+33 n b^{2}+19 n\right) s_{0} \beta+2\left(14 b^{2}-22\right) s_{0} \beta\right] \beta^{2} \\
& M_{15}:=\left[(n+1)\left[4\left(23 b^{4}+5 b^{2}-1\right) c \beta^{2}-\left(14 b^{2}+1\right)\left(2 b^{2}+1\right) \eta \beta\right]\right. \\
& -\frac{n+1}{2}\left(8 b^{2}+1\right) r_{00}-2 \lambda\left(14 b^{2}+1\right)\left(2 b^{2}+1\right)\left(s_{0}+r_{0}\right) \beta \\
& \left.-2\left(-6 b^{2}+3-5 n b^{2}\right) s_{0} \beta-2\left(4+14 b^{2}\right) r_{0} \beta\right], \\
& M_{16}:=(n+1)\left(1+2 b^{2}\right)^{2} c .
\end{aligned}
$$

The term of (25) which is seemingly does not contain $\alpha^{2}$ is $M_{1}$. Since $\beta^{15}$ is not divisible by $\alpha^{2}$, then $c=0$ which implies that

$$
M_{1}=M_{9}=0 \text {. }
$$

Therefore (25) reduces to following

(26) $\quad M_{2}+M_{3} \alpha^{2}+M_{4} \alpha^{4}+M_{5} \alpha^{6}+M_{6} \alpha^{8}+M_{7} \alpha^{10}+M_{8} \alpha^{12}=0$,

(27) $\quad M_{10}+M_{11} \alpha^{2}+M_{12} \alpha^{4}+M_{13} \alpha^{6}+M_{14} \alpha^{8}+M_{15} \alpha^{10}+M_{16} \alpha^{12}=0$. 
By plugging $c=0$ in $M_{2}$ and $M_{10}$, the only equations that don't contain $\alpha^{2}$ are the following

$$
\begin{aligned}
8\left[8\left(2 \lambda\left(r_{0}+s_{0}\right)-(n+1) \eta\right)+6 n r_{00}\right] & =\tau_{1} \alpha^{2}, \\
6\left[48\left(2 \lambda\left(r_{0}+s_{0}\right)-(n+1) \eta\right)+(34 n-1) r_{00}\right] & =\tau_{2} \alpha^{2},
\end{aligned}
$$

where $\tau_{1}=\tau_{1}(x)$ and $\tau_{2}=\tau_{2}(x)$ are scalar functions on $M$. By eliminating $\left[2 \lambda\left(r_{0}+s_{0}\right)-(n+1) \eta\right]$ from $(28)$ and $(29)$, we get

$$
r_{00}=\tau \alpha^{2},
$$

where $\tau=\frac{\tau_{2}-\tau_{1}}{-(18 n+1)}$. By (28) or (29), it follows that

$$
2 \lambda\left(r_{0}+s_{0}\right)-(n+1) \eta=0 .
$$

By (30), we have $r_{0}=\tau \beta$. Putting (30) and (31) in $M_{10}$ and $M_{11}$ yield

$$
M_{10}=(204 n-6) \tau \alpha^{2} \beta^{12},
$$

$$
M_{11}=\left[\left[(660 n-230) s_{0}-660 r_{0}\right] \beta-\frac{(300 n-120) b^{2}+249-189 n}{2} r_{00} \tau \alpha^{2}\right] \beta^{9} .
$$

By putting (32) and (33) into (27), we have

$$
\begin{aligned}
& {\left[(660 n-230) s_{0}-660 r_{0}\right] \beta^{10}-\frac{300 n b^{2}+249-189 n-120 b^{2}}{2} r_{00} \tau \alpha^{2} \beta^{9} } \\
& +(204 n-6) \tau \beta^{12}-M_{12} \alpha^{2}+M_{13} \alpha^{4}+M_{14} \alpha^{6}+M_{15} \alpha^{8}+M_{16} \alpha^{10}=0 .
\end{aligned}
$$

The only equations of (34) that do not contain $\alpha^{2}$ is $\left[(204 n-6) \tau \beta^{2}+(660 n-\right.$ $\left.230) s_{0}-660 r_{0}\right] \beta^{10}$. Since $\beta^{10}$ is not divisible by $\alpha^{2}$, then we have

$$
\left[(204 n-6) \tau \beta^{2}+(660 n-230) s_{0}-660 r_{0}\right]=0 .
$$

By Lemma 3.1, we always have $s_{j}=0$. Then (35), reduces to following

$$
(204 n-6) \tau \beta^{2}-660 r_{0}=0 .
$$

Thus

$$
2(204 n-6) \tau b_{i} \beta-660 \tau b_{i}=0 .
$$

By multiplying (37) with $b^{i}$, we have

$$
\tau=0 \text {. }
$$

Thus by (31), we get $\eta=0$ and then $\mathbf{S}=(n+1) c F$. By $(30)$, we get $r_{i j}=0$. Therefore Lemma 3.1, implies that $\mathbf{S}=0$. This completes the proof. 
Proof of Theorem 1.1. Let $F$ be an isotropic Berwald metric (3) with almost isotropic flag curvature (1). In [19], it is proved that every isotropic Berwald metric (3) has isotropic S-curvature (2).

Conversely, suppose that $F$ is of isotropic S-curvature (2) with scalar flag curvature $\mathbf{K}$. In [10], it is showed that every Finsler metric of isotropic Scurvature (2) has almost isotropic flag curvature (1). Now, we are going to prove that $F$ is a isotropic Berwald metric. In [3], it is proved that $F$ is an isotropic Berwald metric (3) if and only if it is a Douglas metric with isotropic mean Berwald curvature (5). On the other hand, every Finsler metric of isotropic S-curvature (2) has isotropic mean Berwald curvature (5). Thus for completing the proof, we must show that $F$ is a Douglas metric. By Proposition 3.2, we have $\mathbf{S}=0$. Therefore by Theorem 1.1 in [10], $F$ must be of isotropic flag curvature $\boldsymbol{K}=\sigma(x)$. By Proposition $3.2, \beta$ is a Killing 1-form with constant length with respect to $\alpha$, that is, $r_{i j}=s_{j}=0$. Then (14), (15) and (16) reduce to

$$
G^{i}-\bar{G}^{i}=\alpha Q s_{0}^{i}, \quad J_{i}=-\frac{\Phi s_{i 0}}{2 \alpha \Delta}, \quad \bar{J}=0 .
$$

By (12), we get

$$
I_{i} b^{i}=\frac{-\Phi}{2 \Delta F}\left(\phi-s \phi^{\prime}\right)\left(b^{2}-s^{2}\right) .
$$

We consider two case:

Case 1. Let $\operatorname{dim} M \geq 3$. In this case, by Schur Lemma $F$ has constant flag curvature and (21) holds. Thus by (38) and (39), the equation (21) reduces to following

$$
\frac{\Phi s_{i 0}}{2 \alpha \Delta} a^{i k} s_{k 0}+\frac{\Phi s_{l 0}}{2 \alpha \Delta}\left(\alpha Q s_{0}^{l}\right)_{. i} b^{i}-\boldsymbol{K} F \frac{\Phi}{2 \Delta}\left(\phi-s \phi^{\prime}\right)\left(b^{2}-s^{2}\right)=0 .
$$

By assumption $\Phi \neq 0$. Thus by (40), we get

$$
s_{i 0} s^{i}{ }_{0}+s_{l 0}\left(\alpha Q s_{0}^{l}\right)_{. i} b^{i}-\boldsymbol{K} F \alpha\left(\phi-s \phi^{\prime}\right)\left(b^{2}-s^{2}\right)=0 .
$$

The following holds

$$
\left(\alpha Q s_{0}^{l}\right)_{. i} b^{i}=s Q s_{0}^{i}+Q^{\prime} s_{0}^{i}\left(b^{2}-s^{2}\right) .
$$

Then (41) can be rewritten as follows

$$
s_{i 0} s_{0}^{i} \Delta-\boldsymbol{K} \alpha^{2} \phi\left(\phi-s \phi^{\prime}\right)\left(b^{2}-s^{2}\right)=0 .
$$

By (11), (23) and (42), we obtain

$$
\begin{aligned}
& {\left[1-\frac{s\left(1+2 s+3 s^{2}\right)}{\left(-1+s^{2}+2 s^{3}\right)}+\frac{2\left(b^{2}-s^{2}\right)(1+3 s)\left(1+s+s^{2}+s^{3}\right)}{\left(-1+s^{2}+2 s^{3}\right)^{2}}\right] s_{i 0} s_{0}^{i}} \\
& -\boldsymbol{K}\left(1+s+s^{2}+s^{3}\right) \alpha^{2}\left[1+s+s^{2}+s^{3}-s\left(1+2 s+3 s^{2}\right)\right]\left(b^{2}-s^{2}\right)=0 .
\end{aligned}
$$


Multiplying (43) with $\left(-1+s^{2}+2 s^{3}\right)^{2} \alpha^{12}$ yields

$$
A+\alpha B=0
$$

where

$$
\begin{aligned}
A= & -\boldsymbol{K} b^{2} \alpha^{14}+\left(2 b^{2}+1\right)\left(\boldsymbol{K} \beta^{2}+s_{i 0} s_{0}{ }_{0}\right) \alpha^{12} \\
& +2\left(3 \boldsymbol{K} b^{2} \beta^{2}+4 s_{i 0} s^{i}{ }_{0} b^{2}-\boldsymbol{K} \beta^{2}-s_{i 0} s^{i}{ }_{0}\right) \beta^{2} \alpha^{10} \\
& -\left(6 \boldsymbol{K} \beta^{2}+11 s_{i 0} s^{i}{ }_{0}+20 \boldsymbol{K} \beta^{2} b^{2}-6 s_{i 0} s^{i}{ }_{0}\right) \beta^{4} \alpha^{8} \\
& -\left(-20 \boldsymbol{K} \beta^{2}+5 \boldsymbol{K} \beta^{2} b^{2}+8 s_{i 0} s^{i}{ }_{0}\right) \beta^{6} \alpha^{6}+\left(\boldsymbol{K} \beta^{10}\right)\left(26 b^{2}+5\right) \alpha^{4} \\
& -2 \boldsymbol{K} \beta^{12}\left(13-4 b^{2}\right) \alpha^{2}-8 \boldsymbol{K} \beta^{14}, \\
B= & -\left(\boldsymbol{K} b^{2} \beta\right) \alpha^{12}+\left(1+8 b^{2}\right)\left(\boldsymbol{K} b^{2} \beta^{2}+s_{i 0} s^{i}{ }_{0}\right) \beta \alpha^{10} \\
& -2\left(3 \boldsymbol{K} b^{2} \beta^{2}-4 s_{i 0} s^{i}{ }_{0} b^{2}+4 \boldsymbol{K} \beta^{2}+5 s_{i 0} s^{i}{ }_{0}\right) \beta^{3} \alpha^{8} \\
& +\left(6 \boldsymbol{K} \beta^{2}-11 s_{i 0} s^{i}{ }_{0}-20 \boldsymbol{K} \beta^{2} b^{2}\right) \beta^{5} \alpha^{6} \\
& +\left(5 \boldsymbol{K} \beta^{9}\right)\left(3 b^{2}+4\right) \alpha^{4}+\left(5 \boldsymbol{K} \beta^{11}\right)\left(-3+4 b^{2}\right) \alpha^{2}-20 \alpha \boldsymbol{K} \beta^{13} .
\end{aligned}
$$

Obviously, we have $A=0$ and $B=0$.

By $A=0$ and the fact that $\beta^{14}$ is not divisible by $\alpha^{2}$, we get $\mathbf{K}=0$. Therefore (43) reduces to following

$$
s_{i 0} s_{0}^{i}=a_{i j} s_{0}^{j} s_{0}^{i}=0 .
$$

Because of positive-definiteness of the Riemannian metric $\alpha$, we have $s_{0}^{i}=0$, i.e., $\beta$ is closed. By $r_{00}=0$ and $s_{0}=0$, it follows that $\beta$ is parallel with respect to $\alpha$. Then $F=\alpha+\beta+\frac{\beta^{2}}{\alpha}+\frac{\beta^{3}}{\alpha^{2}}$ is a Berwald metric. Hence $F$ must be locally Minkowskian.

Case 2. Let $\operatorname{dim} M=2$. Suppose that $F$ has isotropic Berwald curvature (3). In [19], it is proved that every isotropic Berwald metric (3) has isotropic S-curvature, $\mathbf{S}=(n+1) c F$. By Proposition 3.2, $c=0$. Then by $(3), F$ reduces to a Berwald metric. Since $F$ is non-Riemannian, then by Szabó's rigidity Theorem for Berwald surface (see [2] page 278), F must be locally Minkowskian.

Acknowledgment. The authors would like to thank the referee's valuable suggestions.

\section{References}

[1] S. Bácsó and M. Matsumoto, On Finsler spaces of Douglas typea generalization of the notion of Berwald space, Publ. Math. Debrecen 51 (1997), no. 3-4, 385-406.

[2] D. Bao, S. S. Chern, and Z. Shen, An Introduction to Riemann-Finsler Geometry, Springer-Verlag, 2000.

[3] X. Chen and Z. Shen, On Douglas metrics, Publ. Math. Debrecen. 66 (2005), no. 3-4, 503-512.

[4] X. Cheng and Z. Shen, Randers metric with special curvature properties, Osaka. J. Math. 40 (2003), no. 1, 87-101. 
[5] X. Cheng and Z. Shen, A class of Finsler metrics with isotropic S-curvature, Israel. J. Math. 169 (2009), 317-340.

[6] X. Cheng, H. Wang, and M. Wang, $(\alpha, \beta)$-metrics with relatively isotropic mean Landsberg curvature, Publ. Math. Debrecen. 72 (2008), no. 3-4, 475-485.

[7] N. Cui, On the S-curvature of some $(\alpha, \beta)$-metrics, Acta. Math. Scientia, Series: A. 26 (2006), no. 7, 1047-1056.

[8] I. Y. Lee and M. H. Lee, On weakly-Berwald spaces of special $(\alpha, \beta)$-metrics, Bull. Korean Math. Soc. 43 (2006), no. 2, 425-441.

[9] M. Matsumoto, Theory of Finsler spaces with $(\alpha, \beta)$-metric, Rep. Math. Phys. 31 (1992), no. $1,43-84$.

[10] B. Najafi, Z. Shen, and A. Tayebi , Finsler metrics of scalar flag curvature with special non-Riemannian curvature properties, Geom. Dedicata. 131 (2008), 87-97.

[11] H. S. Park and E. S. Choi, On a Finsler spaces with a special $(\alpha, \beta)$-metric, Tensor (N.S.) 56 (1995), no. 2, 142-148.

[12] Finsler spaces with an approximate Matsumoto metric of Douglas type, Comm. Korean. Math. Soc. 14 (1999), no. 3, 535-544.

[13] Finsler spaces with the second approximate Matsumoto metric, Bull. Korean. Math. Soc. 39 (2002), no. 1, 153-163.

[14] H. S. Park, I. Y. Lee, and C. K. Park, Finsler space with the general approximate Matsumoto metric, Indian J. Pure. Appl. Math. 34 (2002), no. 1, 59-77.

[15] H. S. Park, I. Y. Lee, H. Y. Park, and B. D. Kim, Projectively flat Finsler space with an approximate Matsumoto metric, Comm. Korean. Math. Soc. 18 (2003), no. 3, 501-513.

[16] Z. Shen, Differential Geometry of Spray and Finsler Spaces, Kluwer Academic Publishers, Dordrecht, 2001.

[17] A. Tayebi and B. Najafi, On isotropic Berwald metrics, Ann. Polon. Math. 103 (2012), no. 2, 109-121.

[18] A. Tayebi, E. Peyghan, and H. Sadeghi, On Matsumoto-type Finsler metrics, Nonlinear Anal. Real World Appl. 13 (2012), no. 6, 2556-2561.

[19] A. Tayebi and M. Rafie Rad, S-curvature of isotropic Berwald metrics, Sci. China Ser. A 51 (2008), no. 12, 2198-2204.

[20] C.-H. Xiang and X. Cheng, On a class of weakly-Berwald $(\alpha, \beta)$-metrics, J. Math. Res. Expos. 29 (2009), no. 2, 227-236.

AKBAR TAYEBI

Department of Mathematics

FACULTY OF SCIENCE

UNIVERSITY OF QOM

QOM. IRAN

E-mail address: akbar.tayebi@gmail.com

Tayebeh Tabatabaeifar

Department of Mathematics

FACUlTy OF SCIENCE

UNIVERSITY OF QOM

QOM. IRAN

E-mail address: t.tabaee@gmail.com

Esmaeil Peyghan

Department of Mathematics

FACUlty of SCIENCE

ARAK UNIVERSITY

38156-8-8349 ARAK. IRAN

E-mail address: epeyghan@gmail.com 